\title{
M odelo experimental de aneurisma sacular de artéria ilíaca comum com pericárdio bovino em suínos
}

\author{
Experimental model of saccular aneurysm of the common iliac artery \\ with bovine pericardium in pigs \\ Luís H enrique Gil França', Adamastor H umberto Pereira², Sílvio C ésar Perini', \\ Rodrigo Argenta ${ }^{3}$, Celso Curcio Aveline ${ }^{4}$, Roseli de O liveira M ollerke, M arcos Eugenio Soares ${ }^{6}$, \\ Fernanda N óbrega ${ }^{6}$, Márcio Poletto Ferreira ${ }^{6}$
}

\begin{abstract}
Resumo
O bjetivo: Desenvolver um novo modelo experimental de pseudoaneurisma sacular de artéria ilíaca comum com o uso de re mendo de pericárdio bovino em suínos.

M étodo: Foram utilizados dois suínos da raça Landrace, com peso de $30 \mathrm{~kg}$ e do sexo feminino. 0 s animais foram submetidos a anestesia geral e a laparotomia com acesso extraperitoneal da aorta infrarenal e artérias ilíacas. Após heparinização sistêmica e pinçamento, foram efetuadas arteriotomia longitudinal e sutura do pericárdio bovino em formato de bolsa previamente confeccionada. Os animais permaneceram confinados por 3 semanas e então foram submetidosa arteriografia para avaliação da perviedade do aneurisma e, posteriormente, sacrificados.

Resultado: 0 s animais sobreviveram ao experimento e apresentaram aneurismas pérvios no momento do sacrifício. N ão houve ocorrência de ruptura de aneurismas.

Conclusão: 0 modelo proposto é viável e tem potencial para ser utilizado no estudo e desenvolvimento de novas próteses endovasculares
\end{abstract}

Palavras-chave: aneurisma aórtico, artérias, modelos animais.

0 aneurisma isolado da artéria ilíaca é aquele que acomete uma ou mais artériasilíacas, sem concomitância de outros aneurismas em outra localização. É uma entidade rara, ea artériailíaca comum éacometida com maior freqüência, seguida da artéria ilíaca interna, com a artéria ilíaca externa sendo raramente afetada por

\footnotetext{
1. Mestre. Cirurgião vascular. Doutorando em Cirurgia, Universidade $\mathrm{Fe}-$ deral do Rio Grande do Sul (UFRGS), Porto Alegre, RS.

2. Professor adjunto, UFRGS. Chefe do Serviço de Cirurgia Vascular, Hospital de Clínicas de Porto Alegre (HCPA), Porto Alegre, RS.

3. Cirurgião vascular. Mestrando em Cirurgia, UFRGS, Porto Alegre, RS 4. Anestesista, Hospital São Lucas, Porto Alegre, RS.

5. Doutora. Professora, Fac. de Veterinária, UFRGS, Porto Alegre, RS.

6. Faculdade de Veterinária, UFRGS, Porto Alegre, RS.
}

Artigo submetido em 26.09.05, aceito em 18.11.05.

\begin{abstract}
O bjective: To develop an experimental model of saccular pseudoaneurysm of the common iliac artery with bovine pericardium in pigs.

M ethod: The animals used were two $30-\mathrm{kg}$ female pigs from the Landrace specimen. The animals were submitted to general anesthesia and laparotomy with extraperitoneal access to infrarenal aorta and the iliac arteries. After systemic heparin and clamping, the iliac artery was opened and the pericardium sutured to the vessel wall. All animals were confined for 3 weeks and were sacrificed after performing an arteriography to verify the patency of the aneurysm.

Result: All animals survived the procedure and the aneurysms were patent. No rupture was detected.

Conclusion: This is a feasible pig model of iliac artery aneurysm with potential for further studies to develop new endovascular prostheses.
\end{abstract}

Key words: aortic aneurysm, arteries, animal models.

razões ainda não compreendidas. A maior parte está associada à doença aterosclerótica, como os aneurismas de aorta, esugere-se que placas de ateroma contribuam enfraquecendo a parede da artéria ${ }^{1}$. A história natural não é bem definida, por serem raros e pela falta de seguimento por métodos de imagem.

0 tratamento cirúrgico está indicado quando 0 diâmetro do aneurismaéde $3 \mathrm{~cm}$. A taxa demortalidade por ruptura está entre $25-57 \%$, enquanto a taxa por cirurgia eletiva é de $5 \% 1$. A abordagem terapêutica do aneurisma de ilíaca ultimamente vem passando por profundas mudanças nos conceitos inicialmente estabelecidospara seu tratamento. Com os experimentosde Parodi em 1991, surgiu um novo método, menos invasivo, de correção dos aneurismas: a cirurgia endovascular ${ }^{2}$. 0 entusiasmo depoder tratar 0 aneurisma da 
aorta e outras artérias de uma forma menos agressiva levou alguns pesquisadores a publicar resultados bem promissores de seus experimentos ${ }^{3}$. Então, surgiu a necessidade do desenvolvimento de modelos experimentais em animais, o mais parecido possível com as características morfológicas e hemodinâmicas do ser humano, para estudo da patogêneseedaterapêuticados aneurismas.

0 objetivo desta pesquisaédesenvolver um modelo experimental de aneurisma de artéria ilíaca em suínos, com remendo de pericárdio bovino.

\section{M aterial e método}

0 trabalho foi realizado na U nidade deExperimentação Animal no Centro de Pesquisas do H ospital de Clínicas de Porto Alegre (CP/HCPA), vinculado à U niversidade Federal de Rio Grande do Sul. 0 projeto foi enviado à Comissão C ientífica e ComitêdeÉtica do Grupo de Pós-graduação e Pesquisa do Hospital de Clínicas de Porto Alegre para apreciação dos comemorativos éticos envolvidos no estudo. 0 projeto foi aprovado para realização da parte experimental pela Comissão de Ética do CP/H CPA.

O sanimaisutilizados foram suínos da raça Landrace, com idade aproximada de 8 semanas, com peso de $30 \mathrm{~kg}$, alimentação independente e fornecidos pelo mesmo produtor. A amostra utilizada neste estudo consistiu de dois animais. $0 \mathrm{~s}$ animais foram alojados em baias com água ad li bitum eal imentados com ração (AVIPAL ${ }^{\circledR}$ ) sem suplementação lipídica adicional, balanceada e adaptada para a idade.

A perviedadedosaneurismasfoi confirmadapela realização de arteriografías seletivas, utilizando um aparelho fixo $\left(G E{ }^{\circledR}\right)$. A arteriografia foi realizada no CP/HCPA.

\section{Variáveis em estudo}

Perviedade da artéria ilíaca dos suínos após a realização do modelo experimental do aneurisma;

Perviedade e biocompatibilidade do aneurisma de remendo de pericárdio bovino.

\section{Técnica anestésica}

O $s$ animais foram submetidos a um jejum alimentar de 12 horas para dieta sólida ede 4 horas para dieta líquida. A técnica utilizada será anestesia geral inal atória, utilizando um sistema fechado com reinalação (modelo HUSKY - CALGIMED ${ }^{\circledR}$ ).
0 estudo foi dividido em duas fases. Todos os animais foram pesados antes da indução pré-anestésica. Em ambas as fases, a anestesia do animal foi realizada de acordo com o protocolo de anestesia geral para suínos do CP/HCPA, sendo utilizada a Tiletamina como medicação pré-anestésica, aplicada por via intramuscular, 5 minutos antes de iniciar 0 procedimento. A via de acesso venosa utilizada para infusão de líquidos e drogas foi a veia marginal da orelha. A anestesia parenteral foi realizada com ketamina e xilasina, e a reposição hídrica foi obtida com solução fisiológica 0,9\% a $20 \mathrm{ml} / \mathrm{kg} /$ hora. A anestesia inalatória foi mantida com isoflurano $2 \%$.

\section{Procedimento cirúrgico}

$\mathrm{N}$ a primeira fase, foi realizada uma incisão paramediana esquerda com dissecção da artéria ilíaca comum esquerda. Foi administrada heparina endovenosa (5.000 U ); após, foram pinçadas a artéria proximal e distal. A seguir, foram realizadas a arteriotomia e sutura deremendo de pericárdio bovino com fio depolipropileno 5.0 em forma de bolsa e fechamento da cavidade com fio de vicril 3.0 (Figura 1).

$\mathrm{N}$ a segunda fase, 21 dias após a primeira, foram realizadas arteriografias para confirmação da perviedade dos aneurismas (Figura 2). 0 s animais foram anestesiados e sacrificados, através de injeção intracardíaca de cloreto de potássio, conforme o protocolo do CP/ HCPA. Asartériasilíacas com os aneurismas de pericárdio bovino foram retiradas em monoblocos para a realização deexameanatomopatológico (para avaliação do grau de endotelização e trombos intramurais).

\section{Preparo do tecido para análise patológica}

0 s tecidos retirados foram fixados com solução de aldeído fórmico a $10 \%$ e posteriormente conservados a $4{ }^{\circ} \mathrm{C}$ por 10 dias. A pós a fixação do segmento ressecado, a artéria ilíaca comum foi excisada e realizada nova macroscopia, descrevendo, se houve, as alterações. 0 s segmentos foram processados e incluídos em blocos de parafina e posteriormente submetidos a cortes histológicos medindo $4 \mu \mathrm{m}$ de espessura. As lâminas contendo os cortes de tecidos foram preparadas e coradas com hematoxilina-eosina.

\section{Resultados}

O s animais sobreviveram ao experimento, e não ocorreram intercorrências clínicas durante o período 
do estudo. 0 saneurismas estavam pérviosno momento do sacrifício. Não houve ocorrência de ruptura de aneurismas. Ao exame, o pericárdio bovino apresentou boa integração à parede do vaso, ausência de trombos murais e endotelização.

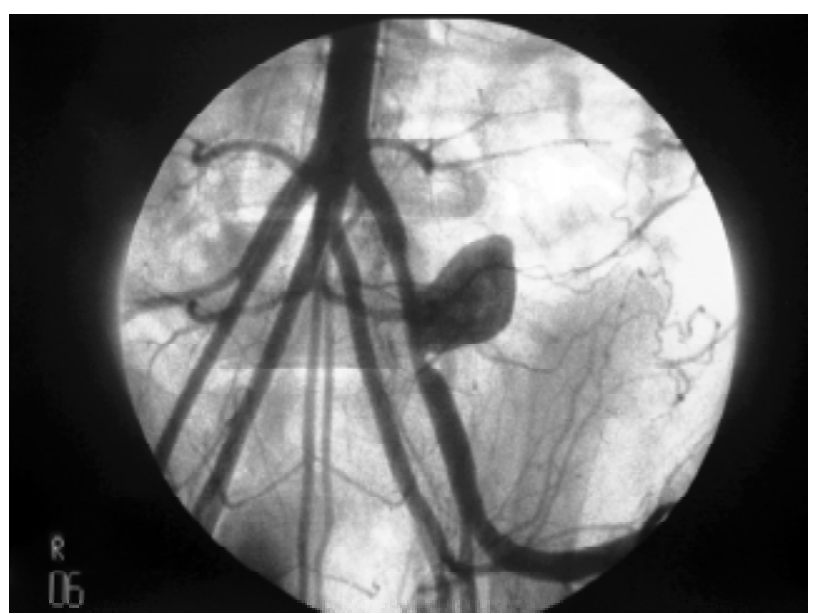

Figura 1 - Arteriografiapós-operatóriademonstrando aneurisma de artéria ilíaca esquerda

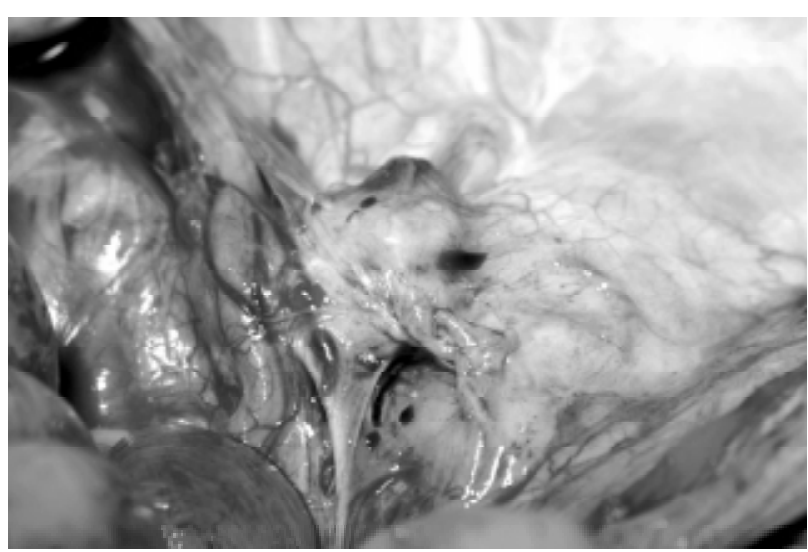

Figura 2 - Peça cirúrgica de aneurisma de artéria ilíaca com pericárdio bovino (exame anatomopatológico)

\section{D iscussão}

Em 1999, W olf et al. estabeleceram que o modelo suíno tem como vantagens o fácil manejo dos vasos de grosso calibre e o fato de o metabolismo lípidico, 0 perfil lipoprotéico e a agregação plaquetária serem semelhantes aos humanos, além de que, após a lesão endotelial, ocorre formação de trombo e deposição de fibrina, de maneira similar com humanos. Todavia, existem desvantagens, como o rápido aumento de peso, o gasto com a manutenção ea suscetibilidade à fibrilação ventricular quando da real ização deoclusão coronária durante um procedimento de angioplastia. $O$ utros animais, como os primatas, apresentam características muito semelhantes aos humanos, conforme as citadas acima, porém apresentam muita sensibilidade, a dieta varia conforme a espécie, são de difícil manuseio, além de os estudos apresentarem um custo muito elevado. Por esses motivos, o suíno foi escolhido como modelo experimental ${ }^{4}$.

Com um modelo experimental introduzido em 1990 por Anidjar et al., foi possível um grande avanço na compreensão da importância do processo inflamatório na gênese e no desenvolvimento dos aneurismas, relacionan do a degradação da elastina esubstituição por fibras de colágeno tipos I ellI como responsáveis pela formação eexpansão dosaneurismas ${ }^{5}$. Boudgheneet al. utilizaram elastase para criar aneurisma de aorta abdominal em cães. As artérias lombares foram ligadas nos dois lados exceto distais ao segmento desejado. A pós a administração de heparina endovenosa e pinçamento do segmento deaorta desejado, foi iniciada a infusão de $2.800 \mathrm{U}$ desolução de elastase porcina tipo I na taxa de $60 \mathrm{ml} / \mathrm{h}$ durante 40 minutos. Todos os animais desenvolveram aneurisma com diâmetro de aproximadamente $100 \%$ acima do valor inicial. 0 examehistológico mostrou a lâmina elástica fragmentada em todas as sessões e desaparecida em al gumasáreas ${ }^{6}$. Essa al teração patológica assemelha-se aos aneurismas de aorta abdominal dehumanos. $\mathrm{N}$ o entanto, aligaduradaslombares não torna esse modelo ideal para testar vazamentos ea tendência à ruptura.

$\mathrm{H}$ allisey criou um aneurisma de aorta infra-renal em cães utilizando técnicas endovasculares para dilatar a aorta cerca de duas vezes o seu diâmetro. Então, foi criado um aneurisma fusiforme com as artérias lombares preservadas ${ }^{7}$. Porém, a morfologia desse aneurisma é estável, sem expansão e sem tendência à ruptura. Pode ser utilizado para treinamento em procedimentos endovasculares.

Vários estudos já desenvolveram modelos de aneurisma de aorta, principal mente em cachorros e porcos. Esses aneurismas foram criados mediante sutura de um remendo de material sintético (PTFE ou Dacron ${ }^{\circledR}$ ), fáscia, peritônio, intestino e também pela interposição de um seguimento de veia jugular interna bovina conservado em glutaraldeído após a ressecção do segmento de aorta infra-renal ${ }^{8-13}$. A vantagem deste último mo- 
delo éa forma. Entretanto, no intuito de estudar novas técnicas endovasculares, esse model o perde a função de avaliar os tipos de vazamentos e de selar a parede da aorta, já que os vasos colaterais não estão pérvios. O s model os que utilizam remendo na parede anterior são bem aceitos, com a principal vantagem de manter a perviedade das artérias lombares. M aynar et al., em estudo de aneurisma de aorta abdominal com o remendo deperitônio, concluíram queessemodelo representa a capacidade de expansão, ruptura e perviedade dos ramos colaterais, sendo um modelo factível para o estudo de endopróteses ${ }^{12}$.

$\mathrm{N}$ o estudo $\mathrm{N}$ ine year experience of bovine pericardium patch angioplasty during carotid endarterectomy, os autores analisam o resultado do uso de remendo de pericárdio bovino preparado com glutaraldeído para arterioplastia pós-endarterectomia de carótida ${ }^{14}$. 0 pericárdio cru é retirado com saco intacto de animais com menos de 1 ano e tratado com solução deglutaraldeído. Após, trata-se com solução de etanol eóxido de propileno para retirar o glutaraldeído residual, já que um resíduo de 4 ppm se mostrou tóxico para os fibroblastos. Este tecido é relativamente acelular, e sua constituição primária éde colágeno tipo I , sofrendo um processo de endotelização. 0 glutaraldeído serve para ligar os grupos de aminas e esterilizá-los. Esse cross linking das moléculas de colágeno mascara os sítios antigênicos e os sítios das proteases de clivagem, retardando a sua degradação. 0 material foi preparado em glutaral deído para diminuir a antigenicidade, fortalecer e aumentar a resistência à degradação. O s remendos periféricos apresentam menores índices de calcificação quando comparados com as vál vulas cardíacas. A dilatação aneurismática no enxerto de pericárdio deve-se à degeneração resultante da antigenicidade. Porém, por ser um material acelular, essa resposta é mínima, não havendo relatos de ruptura como há com próteses de veia ou material sintético ${ }^{15-17}$.

$\mathrm{N}$ o presente estudo, o desenvolvimento do modelo proposto é viável e tem potencial para ser utilizado no estudo e desenvolvimento de novas próteses endovasculares. T rata-se de modelo único queutiliza material biocompatível de baixo custo. 0 uso de pericárdio bovino, além das vantagens econômicas, apresenta facilidade de manuseio pelo cirurgião e características muito semelhantes ao tecido autólogo na sua integração à parede vascular. N ão há relato na literatura sobre a realização desse modelo com remendo de pericárdio bovino.

\section{Referências}

1. França $L H$, Bredt $C G, B$ ack $L A$, et al. T ratamento cirúrgico do aneurisma isolado da artéria ilíaca. Rev Angiol Cir Vasc. 2002;11:166-71.

2. ParodiJC. Endovascular repair of abdominal aorticaneurysms and other lesions. J V asc Surg. 1995;21:549-57.

3. Quinn SF, Sheley RC, Semonsen KG, et al. Endovascular stents covered with pre-expanded polytetrafluoroethylele for treatment of iliac artery aneurysms and other fistulas. J V asc Interv Radiol. 1997;8:1057-63.

4. W olf YG, G ertz D, B anai S. Animal models in syndromes of accelerated arteriosclerosis. Ann V asc Surg. 1999;13:328-38.

5. Anidjar S. Salzmann JL, G entric D, et al. Elastase-induced experimental aneurysmsin rats. C irculation. 1990;82:973-81.

6. Boudghene FP, Sapoval M R, Bonneau M, et al. Abdominal aortic aneurysms in sheep: prevention of rupture with endoluminal stent-grafts. Radiology. 1998;206:447-54.

7. H allisey M J. 1997 SC VIR Gary J. Becker Young I nvestigator Award Paper. A transluminally created abdominal aortic aneurysm model. J V asc Interv Radiol. 1997;8:305-12.

8. Wisselink W, Abruzzo FM, Shin CK, et al. Endoluminal repair of aneurysmscontaining ostia of essential branch arteries: an experimental model. J Endovasc Surg. 1999;6:171-9.

9. Lerouge S, Raymond J, Salazkin I, et al. Endovascular aortic aneurysm repair with stent-grafts: experimental models can reproduce endoleaks. J V asc Interv Radiol. 2004;15:971-9.

10. D iaz S, U zieblo M R, D esai $K M$, et al. Type II endoleak in porcine model of abdominal aortic aneurysm. J Vasc Surg. 2004;40:339-44.

11. SoulaP, Janned' $O$ theeB, $O$ tal $P$, et al. $M$ acroporouspolyestercovered stent in an experimental abdominal aortic aneurysm model. J Endovasc Ther. 2001;8:390-400.

12. M aynar M, Q ian Z, H ernandez J, et al. An animal model of abdominal aortic aneurysm created with peritoneal patch: technique and initial results. Cardiovasc Interv Radiol. 2003;26:168-76.

13. Faries $P L$, Sanchez $L A, M$ arin $M L$, et al. An experimental model for theacuteand chronic evaluation of intra-aneurysmal pressure. J Endovasc Surg. 1997;4:290-7.

14. W hitebread T, Birch P, Rogers S, et al. A new animal model for abdominal aortic aneurysms: initial resultsusingamultiplewire stent. Eur J V asc Endovasc Surg. 1996;11:90-7.

15. Biasi GM, Sternjakob S, M ingazzini PM, et al. N ine year experience of bovine pericardium patch angioplasty during carotid endarterectomy J V asc Surg. 2002;36:271-7.

16. Anacleto JC, Sanchez RR, M ingardi AL. Bioprótese de pericárdio bovino como substituto arterial no tratamento do aneurisma de aorta abdominal. Cir Vasc Angiol. 1993;9(Supl):35.

17. Salles CA, Puig IS, Casagrande GL, et al. Early experience with crimped bovine pericardial conduit for arterial reconstruction. Eur J Cardiovasc Surg. 1991;5:273.

Correspondência:

Luís H enrique G il França

Rua C oronel Dulcídio, 1189/1801

CEP 80250-100 - Curitiba, PR

Tel.: (41) 3343.0963

E-mail: luishgf@hotmail.com 